\section{SAT0265 DETERMINANTS OF RENAL RESISTIVE INDEX (RRI) AT RENAL ARTERY DOPPLER ULTRASOUND IN SYSTEMIC SCLEROSIS (SSC): MORE THAN GENERAL POPULATION FACTORS}

Cosimo Bruni ${ }^{1}$, Edoardo Rosato ${ }^{2}$, Antonietta Gigante ${ }^{2}$, Vanessa Maestripieri ${ }^{1}$, Giulia Tesei ${ }^{1}$, Marco Chiostri ${ }^{1}$, Gemma Lepri ${ }^{1}$, Silvia Bellando Randone ${ }^{1}$, Serena Guiducci ${ }^{1}$, Sergio Castellani ${ }^{1}$, Maria Boddi $^{1}$, Marco Matucci-Cerinic ${ }^{1}$. ${ }^{1}$ Careggi University Hospital, Firenze, Italy, ${ }^{2}$ Sapienza University of Rome, Roma, Italy

Background: RRI can be measured on renal artery Doppler ultrasound, showing the difficulty blood flow encounters distally from where it is measured. RRI can be determined by vascular and interstitial renal features: studies on the general population showed arterial hypertension, arteriosclerosis, low-grade inflammation, diabetes mellitus, hyperuricaemia as possible causes of renal vasculopathy or tubular-interstitial nephropathy, thus determining altered RRI values. Studies on SSc have shown increased RRI values, associated with nailfold-videocapillaroscopy pattern and history of digital ulcers.

Objectives: to identify determinants of RRI in SSc patients.

Methods: SSc patients fulfilling the 2013 ACR/EULAR criteria were enrolled from two SSc-care units. Data regarding SSc clinical manifestations, instrumental and laboratory evaluation for renal, cardiac and cardiovascular involvement were collected. RRI was measured at least once in each patient. Linear regression analysis was carried out to determine predictors for baseline $R R I$ and change in $R R I$ in time $(\Delta \mathrm{RRI})$.

Results: 380 patients [aged 57 (46-68) years, 12\% males] were enrolled in the study. On univariate analysis, both general population determinants (age, arterial hypertension, diabetes mellitus, hyper-lipidaemia and hyper-uricaemia) and SSc specific features [time from Raynaud's phenomenon onset, ACA positivity, scleroderma renal crisis history, estimates systolic pulmonary arterial pressure on Echo (sPAP), presence of dyspnea,\%DLCO and presence of lower intestinal symptoms] predicted RRI values, with only age and SPAP being significant independent predictors at baseline. Follow-up RRI, available for 230 patients, showed that none of the general population determinants was predictive for $\triangle R R I$, while presence of telangectasias, baseline FVC, use of Sildenafil, change in SPAP and new diagnosis of PAH were predictive at univariate regression. Among them, only presence of baseline telangectasias was an independent predictor of $\Delta R R I$ at multivariate regression analysis.

Conclusion: Data show that among general population determinants, age is the only predictive factor for RRI in SSc patients. However, also SPAP (for RRI) and telangectasias could be predicitive for $\Delta$ RRI. Therefore, the use of specific age-adjusted cut-offs (table 1) are recommended when assessing SSc patients.

Data show that among general population determinants, age is the only predictive factor for RRI in SSc patients. However, also SPAP (for RRI) and telangectasias could be predicitive for $\triangle R R I$. Therefore, the use of specific age-adjusted cut-offs (table 1) are recommended when assessing SSc patients.

Table 1. Age-adjusted cut-offs for Renal Resistive Index (RRI) in Systemic sclerosis patients.

\begin{tabular}{lc}
\hline Age (years) & Age-SSc adjusted Pathologic RRI \\
\hline 1st quartile $\leq 49$ y & $\geq 0.68$ \\
2nd quartile $50-59$ y & $\geq 0.70$ \\
3rd quartile $60-69$ y & $\geq 0.75$ \\
4th quartile $\geq 70$ y & $\geq 0.78$ \\
\hline
\end{tabular}

Disclosure of Interests: Cosimo Bruni: None declared, Edoardo Rosato: None declared, Antonietta Gigante: None declared, Vanessa Maestripieri: None declared, Giulia Tesei: None declared, Marco Chiostri: None declared, Gemma Lepri: None declared, Silvia Bellando Randone: None declared, Serena Guiducci: None declared, Sergio Castellani: None declared, Maria Boddi: None declared, Marco Matucci-Cerinic Grant/ research support from: Actelion, MSD, Pfizer, BMS, Chemomab, Sanipedia, Speakers bureau: Actelion, BMS; MSD, Janssen

DOI: 10.1136/annrheumdis-2019-eular.3514

\section{SAT0266 DIGITAL ULCER (DU) AND VENTRICULAR ARRHYTHMIAS PREDICT THE LATE GADOLINIUM ENHANCEMENT (LGE) IN CARDIAC MAGNETIC RESONANCE (CMR) IN SYSTEMIC SCLEROSIS (SSC): PROPOSAL OF CANDIDATE RED FLAGS FOR EARLY REFERRAL}

Cosimo Bruni ${ }^{1}$, Luna Gargani ${ }^{2}$, Alessia Pepe ${ }^{3}$, Antonella Meloni ${ }^{3}$, Daniele De Marchi $^{3}$, Giancarlo Todiere ${ }^{3}$, Gennaro D'angelo ${ }^{2}$, Alberto Moggi Pignone ${ }^{1}$, Serena Guiducci ${ }^{1}$, Marco Matucci-Cerinic ${ }^{1}{ }^{1}$ Careggi University Hospital, Firenze, Italy; ${ }^{2}$ CNR IFC Istituto di Fisiologia Clinica, Pisa, Italy; ${ }^{3}$ Fondazione Toscana "Gabriele Monasterio", Pisa, Italy

Background: in SSc, cardiac involvement either primary or secondary, is frequently subclinical. In myocardial diseases, CMR is considered the non-invasive gold standard to diagnose myocardial tissue involvement and LGE has a significant impact on prognosis.

Objectives: to identify the predictors of the presence of LGE in SSc patients. Methods: patients fulfilling the 2013 ACR/EULAR classification criteria were assessed with CMR (1.5 T GE scanner) when clinically indicated. Data on demographics, clinical SSc features, laboratory and instrumental tests for cardio-pulmonary, microvascular and renal involvements were recorded at the time of CMR evaluation. Binary regression analysis was performed with SPSS version 20.0 .

Results: 317 SSc patients (284 women, mean age 52 14 ) were enrolled in the study. LGE-CMR was present in $32.9 \%$ and Myocardial edema (ME-CMR) in $6.1 \%$ of patients. On univariate regression analysis,\%DLco values, NYHA functional class, history of DU (hDU), TAPSE and couples of ventricular premature beats (VPBC) were predictors of LGE-CMR or ME-CMR. At multivariate analysis,TAPSE (OR $0.832,95 \% \mathrm{Cl} 0,727-0,951$, $\mathrm{p}=0.007$ ), hDU (OR 3.893, 95\% Cl 1.614-9.387, $\mathrm{p}=0.002$ ) and VPBc (OR $4.238,95 \% \mathrm{Cl} 0.959-18.738, \mathrm{p}=0.057$ ) were independent predictors for LGE-CMR. Presence of pericardial effusion was the only predictor for ME-CMR (OR 3.379, 95\% Cl 1.018-12.988, $\mathrm{p}=0.047$ ).

Conclusion: DU history, ventricular ectopic beats on 24 hours ECG TAPSE impairment and pericardial effusion on echocardiography may predict myocardial LGE. More studies are necessary to further verify and validate these "red flags" to refer the patients to CMR.

Disclosure of Interests: : Cosimo Bruni: None declared, Luna Gargani: None declared, Alessia Pepe: None declared, Antonella Meloni: None declared, Daniele De Marchi: None declared, Giancarlo Todiere: None declared, Gennaro D'Angelo: None declared, Alberto Moggi Pignone: None declared, Serena Guiducci: None declared, Marco Matucci-Cerinic Grant/research support from: Actelion, MSD, Pfizer, BMS, Chemomab, Sanipedia, Speakers bureau: Actelion, BMS; MSD, Janssen DOI: 10.1136/annrheumdis-2019-eular.5618

\section{SAT0267 SERUM ANTI-HETEROGENEOUS NUCLEAR RIBONUCLEOPROTEIN ANTIBODY CHARACTERIZES SYSTEMIC SCLEROSIS WITH ANTI CENTROMERE ANTIBODY AND SEVERE RAYNAUD'S PHENOMENON WITH DIGITAL ULCERS}

Natasa Isailovic ${ }^{1},{ }^{1,2}$ Angela Ceribelli, Carolina Gorlino ${ }^{1}$, Elena Generali ${ }^{1}$, Maria De Santis ${ }^{1}$, Marta Caprioli ${ }^{1}$, Giacomo Maria Guidelli ${ }^{1}$, Piercarlo Sarzi-Puttini ${ }^{2,3}$ Minoru Satoh ${ }^{4}$, Carlo Selmi ${ }^{1,3} .{ }^{1}$ Humanitas Clinical and Research Hospital, Rheumatology and Clinical Immunology, Rozzano (MI), Italy; ${ }^{2}$ Luigi Sacco University Hospital, Rheumatology, Milan, Italy; ${ }^{3}$ University of Milan, Milan, Italy, ${ }^{4}$ University of Occupational and Environmental Health, Clinical Nursing, School of Health Sciences, Kitakyushu, Japan

Background: Systemic Sclerosis (SSc) is characterized by the presence of serum autoantibodies (autoAbs) which may be crucial in the diagnosis, prediction of organ involvement, follow-up, and treatment choices. However, SSc sera can be negative for autoAbs identified using commercially available tests, and several new and rare autoAbs have been identified in the last decade with immunoprecipitation (IP) techniques. We recently reported a new IP pattern corresponding to anti-heterogeneous nucla ribonucleoprotein (hnRNP) antibodies in a cohort of SSc patients

Objectives: To analyze the IP pattern of anti-hnRNP antibodies in SSc sera and determine their clinical and laboratory correlations.

Methods: We investigated sera from 63 consecutive patients with SSc attending our Unit between 2014 and 2018, using protein-IP of ${ }^{35} \mathrm{~S}$ methionine-labeled K562 cell extract followed by SDS-PAGE and autoradiography, and IP-Western Blot for hnRNP-L and C1+C2 following 